\title{
Stress, Depressão e Qualidade de Vida em Beneficiários de Programas de Transferência de Renda
}

\author{
Mariani da Costa Ribas-Prado ${ }^{1}$ \\ Universidade Estadual Paulista Júlio de Mesquita Filho - Bauru \\ Sandra Leal Calais ${ }^{2}$ \\ Universidade Estadual Paulista Júlio de Mesquita Filho - Bauru \\ Hugo Ferrari Cardoso ${ }^{3}$ \\ Universidade Estadual Paulista Júlio de Mesquita Filho - Bauru
}

\begin{abstract}
RESUMO
O stress, como resposta a situações aversivas, pode ser associado à condição de baixo poder aquisitivo. Também a depressão, enquanto um transtorno que compromete a relação do indivíduo com seu ambiente, muitas vezes se relaciona a situações de risco socioeconômico. Ambos referem uma baixa qualidade de vida. No presente estudo investigou-se a prevalência de stress e depressão e a percepção da qualidade de vida em beneficiários de programas de transferência de renda. Utilizou-se o Inventário de Sintomas de Stress para Adultos de Lipp, o Inventário de Depressão de Beck, o Instrumento Abreviado de Avaliação da Qualidade de Vida e um Questionário Sociodemográfico. Os dados foram coletados em 198 participantes, em sua maioria mulheres, sendo que 58\% apresentaram stress e $41 \%$ indicativo de depressão. A percepção média de qualidade de vida variou de 43,0 no domínio meio ambiente a 54,9 no psicológico, em uma escala que vai de 0 a 100. Os resultados sinalizam que na amostra dos beneficiários de transferência de renda, o stress se apresentou não muito diferente de outras amostras da população. No entanto, quanto ao indicativo de depressão e percepção de qualidade de vida este grupo demonstrou resultados mais desfavoráveis do que pessoas em geral. Stress, depressão e qualidade de vida se mostraram correlacionados.
\end{abstract}

Palavras-chave: Stress; depressão; qualidade de vida; vulnerabilidade social.

\section{ABSTRACT \\ Stress, depression and quality of live in beneficiaries of social programs}

Stress, in response to aversive situation, can be associated to poverty condition. Also depression, while a disorder that affects the relationship of the individual with environment, often related to socioeconomic difficulties. Both refer to a low quality of life. This study aimed to investigate the prevalence of stress and depression and the perception of quality of life in beneficiaries of social programs. Data were collected with 198 participants, mostly women, and 58\% had stress and $41 \%$ indicative of depression. The average perception of quality of life ranged from 43.0 in the environment domain to 54.9 in the psychological, in a scale from 0 to 100 . The results indicate that the sample didn't have more stress than the people in general. However, this group showed indication of depression and perception of quality of life more unfavorable. Stress, depression and quality of life were correlated.

Keywords: Stress, depression, quality of life, social vulnerability.

O conceito de stress foi introduzido na área da saúde, em 1926, pelo médico austríaco Hans Selye para designar a Síndrome Geral de Adaptação, a qual é ocasionada por diferentes agentes noviços que debilitam o organismo, enfraquecendo-o e o levando ao adoecimento (Lipp, 1996; Pafaro \& Martino, 2004). Selye, em 1956, descreveu esta síndrome salientando que o processo de stress se desenvolve por meio de três fases: alerta, resistência e exaustão (Alchieri \& Cruz, 2004).

A fase de Alerta se caracteriza pelo confronto inicial do indivíduo com um estressor. Quando a duração do estressor é curta, a adrenalina é eliminada e o indivíduo retorna à homeostase, saindo desta fase sem complicações para seu bem-estar. Porém, quando o estressor é de longa duração ou muito intenso, a pessoa tenta restabelecer a homeostase de um modo reparador, entrando na fase de resistência. Nesta fase, o organismo pode enfraquecer e ficar vulnerável a doenças. Se sua resistência não for suficiente para lidar com a fonte de stress, ou se outros estressores ocorrerem simultaneamente, o pro- 
cesso de stress evoluirá para a próxima fase, a fase de exaustão, que é caracterizada pelo reaparecimento dos sintomas da fase de alerta tais como aumento da frequência cardíaca e respiratória e da pressão arterial e maior concentração de glóbulos vermelhos, brancos e de açúcar no sangue. Pode ocorrer esgotamento físico e psicológico devido à exaustão psicofisiológica e aparecerem doenças graves que ocasionalmente podem levar à morte (Lipp, 1996; Pafaro \& Martino, 2004).

Outro autor sugeriu uma quarta fase ao processo de stress: a fase de quase-exaustão, que acontece entre as fases de resistência e de exaustão. Nesta fase, ocorre um enfraquecimento do indivíduo que não consegue se adaptar ou resistir ao estressor. Surgem doenças relacionadas aos órgãos mais vulneráveis naquele indivíduo, porém estas não são tão graves como na fase de exaustão. O acréscimo de uma fase entre a resistência e exaustão foi proposto durante pesquisas relacionadas à padronização do Inventário de Sintomas de Stress para Adultos de Lipp. Segundo Lipp (2000), esta nova fase foi identificada tanto na clínica como estatisticamente. A autora enfatiza que os dados mostraram que a fase de resistência da forma proposta por Selye era muito ampla, apresentando dois momentos diferenciados pela quantidade e intensidade dos sintomas.

A presença de stress tem sido associada a variáveis sociodemográficas em diversas pesquisas com diferentes populações. Estudos realizados revelam que sexo feminino, baixa escolaridade, não ter filhos, ser solteiro e menor renda familiar frequentemente se relacionam a índices de stress mais elevados (Dantas, Brito, Rodrigues, \& Maciente, 2010; Lipp, 2000; Ramos \& Ferreira, 2011; Sparrenberger, Santos, \& Lima, 2004). Outras pesquisas enfatizam a relação entre variáveis culturais, como pobreza e instabilidade familiar, e o desenvolvimento do stress (Dressler \& Balieiro, 2002; Kaplan, Madden, Mijanovich, \& Purcaro, 2013; Prigerson, Maciejewski, \& Rosenback, 2002).

De forma mais detalhada, Sparrenberger et al. (2004) realizaram estudo com 3.942 pessoas com idade superior a 20 anos, em um município de uma cidade do sul do Brasil, cujos objetivos foram investigar o efeito dos eventos produtores de stress, como morte de alguém significativo, doença em familiar e separação conjugal. A frequência de stress na amostra foi de $14 \%$ e associou-se significativamente a todas as variáveis sociodemográficas estudadas, em que as mulheres apresentaram prevalência maior do que os homens e os não-brancos maior do que a dos brancos. Observou-se também que pessoas na faixa etária de 50 a 59 anos apresentaram prevalência cerca de duas vezes maior do que os indivíduos de 20 a 29 anos. Também foi encontrada associação inversa entre renda e escolaridade, ou seja, quanto maior a escolaridade ou a renda apresentada, menor a frequência de stress observada.

Rodríguez, Martínez, Linares, Burón, López e Guadalupe (2008) ressaltaram que indivíduos com hábitos pouco saudáveis (como fumar, consumir bebida alcoólica e sedentarismo) mostram maior probabilidade de desenvolverem stress frente a situações estressoras. $\mathrm{O}$ stress, por sua vez, proporciona um enfraquecimento de seu sistema imunológico, podendo ocasionar problemas de saúde. Para os autores, a queda do sistema imunológico, somada aos hábitos pouco saudáveis, contribui para a não resolutividade da situação estressora, ocasionando um ciclo que pode culminar com o adoecimento do indivíduo, vindo a exibir uma doença crônica física como hipertensão arterial ou até mesmo um transtorno mental como quadros psicóticos e depressivos.

Neste sentido, Sparrenberger et al. (2004) afirmaram que eventos produtores de stress podem desencadear problemas de saúde que vão desde um resfriado comum a transtornos mentais como esquizofrenia e depressão. Prigerson, Maciejewiski e Rosenback (2002) acreditam que isso acontece quando os indivíduos que experenciam esses eventos, em função do desgaste físico e psíquico envolvidos, não os superam em um determinado período de tempo. Os autores apontam a relevância de estudos que procuram identificar a relação entre stress e os transtornos mentais.

Segundo dados da Organização Mundial de Saúde (OMS) de 2014, a depressão se constituiu como um dos transtornos mentais de maior prevalência na população mundial, atingindo cerca de 350 milhões de pessoas (Munhoz, Nunes, Wehrmeister, Santos, \& Matijasevich, 2016). Martin, Quirino e Mari (2007) enfatizam que o transtorno depressivo se caracteriza como uma desordem mental que compromete o relacionamento do indivíduo com o ambiente em diferentes âmbitos, como família, trabalho e comunidade, estabelecendo-se como um grave problema de saúde pública.

Conforme Máximo (2010), algumas pesquisas epidemiológicas têm sido realizadas no Brasil a respeito da depressão que apontam os seguintes aspectos em relação à sua prevalência: é duas vezes mais frequente em mulheres do que em homens; parece não haver diferenças significativas entre os sexos quanto ao início da de- 
pressão, que tem sido em torno de 24 anos de idade; independente do sexo, a depressão costuma ser mais prevalente entre os indivíduos de menor renda, com menor escolaridade, desempregados e que passaram por separação conjugal recente; o stress associado a esses eventos tem sido colocado como um de seus desencadeantes.

Com base em levantamento realizado em 18 países durante o ano de 2009, a OMS ressalta que $14,6 \%$ da população dos países com alto rendimento socioeconômico já tiveram depressão. Entre o grupo de países com renda baixa e média, $11,1 \%$ das pessoas manifestaram o transtorno em algum momento da vida. A prevalência registrada no Brasil foi de 10,4\%. Os pesquisadores observaram que nos países mais desenvolvidos a idade média para início dos episódios depressivos foi de 25,7 anos contra 24 anos nos países mais pobres. Porém, nos países com alta renda os jovens foram considerados o grupo mais vulnerável ao desenvolvimento do transtorno, enquanto nos demais países foi observada maior vulnerabilidade entre os idosos. Em todos os grupos, separação de um parceiro se mostrou como fator mais relevante, a ocorrência foi duas vezes maior em mulheres e a incapacitação funcional se mostrou associada a manifestações recentes do transtorno (Bromet et al., 2011).

O estudo de (2009) sobre a prevalência dos sintomas depressivos em 954 mexicanos que se encontravam em desvantagem social devido à pobreza procurou relacionar os sintomas depressivos às condições vivenciadas por esta população. Os autores verificaram que $35 \%$ dos homens e $50 \%$ das mulheres exibiram sintomas significativos de depressão, que foram associados às dificuldades da vida diária. Não foi encontrada associação entre o nível de pobreza e os sintomas depressivos e os autores refletiram que isto pode ser explicado pela falta de variabilidade na renda, pois todos os participantes estavam inscritos em um Programa cujo objetivo é combater a pobreza.

Cunha, Bascos e Del Duca (2012), em investigação com adultos e idosos em uma comunidade de baixa renda de Porto Alegre, Rio Grande do Sul, apontaram prevalência de depressão de $16,1 \%$, associada à diminuição dos níveis de escolaridade e renda. No mesmo sentido, Lund et al. (2011), ao analisarem o impacto de programas de transferência condicionada de renda na saúde mental de seus beneficiários, afirmaram que pobreza se constitui como fator de risco para o desenvolvimento de doenças mentais.
Segundo Santos (2011), o ambiente sociocultural tem grande influência na prevalência da depressão. Este autor enfatiza que o número de estudos realizados com a população de baixa renda no Brasil é muito pequeno quando comparado à quantidade de pesquisas feitas com as classes economicamente mais favorecidas. Para ele, ao negligenciar esta população, contribui-se com o aumento da prevalência de sintomas depressivos, e, consequentemente, com a diminuição dos índices de qualidade de vida.

O conceito de qualidade de vida é subjetivo, multidimensional e inclui dimensões positivas e negativas. Engloba visão de mundo e percepções em relação aos aspectos físicos, psicológicos e sociais do indivíduo. Desta forma, qualidade de vida diz respeito à percepção de um indivíduo sobre a sua posição na vida, na cultura em que vive e os sistemas de valores em que acredita, relacionados às suas expectativas, objetivos e inquietações (The Whoqol Group, 1995).

Para Gordia, Quadros, Campos e Vilela-Junior (2009), a qualidade de vida de uma população se relaciona às suas condições de existência e a seu acesso a determinados bens e serviços econômicos e sociais. Estes autores enfatizam a importância do acesso ao emprego e renda, à educação básica, à alimentação adequada, a bons serviços de saúde, ao saneamento básico, à habitação e ao transporte na determinação dos índices de qualidade de vida.

Blay e Marchesoni (2011) mencionam a influência de determinados fatores na avaliação da qualidade de vida. Para estes autores, variáveis socioeconômicas, presença de comorbidades, idade, apresentação de sintomas depressivos e/ou psicóticos, tentativas de suicídio e dor crônica se relacionam aos escores obtidos na avaliação da qualidade de vida.

Várias pesquisas têm sido realizadas com diferentes populações com o intuito de se verificar a qualidade de vida (Baptista, Vargas, \& Baptista, 2008; Blay \& Marchesoni, 2011; Gordia et al., 2009; Mastropietro, Oliveira-Cardoso, Simões, Voltarelli, \& Santos, 2010; Nunes \& Santos, 2010; Pereira, Alvarez, \& Traebert, 2011). Blay e Marchesoni (2011) realizaram um estudo sobre a avaliação da qualidade de vida com universitários de meia idade e universitários idosos e observaram relação significativa entre os índices de qualidade de vida e os indicativos de depressão, sendo que maiores escores de qualidade de vida foram associados a menores índices de depressão.

Pereira et al. (2011) examinaram 141 idosos com o 
objetivo de verificar a relação das condições sociodemográficas com a autoavaliação da qualidade de vida. Os autores apontaram que no domínio meio ambiente, renda familiar inferior a três salários mínimos e a escolaridade inferior a oito anos de estudo se mostraram significativamente associadas a piores índices de qualidade de vida. No domínio físico, além da renda inferior a três salários mínimos e da escolaridade inferior a oito anos de estudo, a condição de não ter companheiro(a) e a idade de 80 anos ou mais também estiveram associadas a escores menores. Para os autores, tais dados indicam que idade avançada se mostra relacionada à pior percepção da qualidade de vida.

Probst, Laditka, Moore, Harun e Powell (2005) e Hernández, Moreno e Borges (2007) enfatizam que a condição socioeconômica é um dos fatores que pode interferir nos índices de qualidade de vida. Assim, possuir baixa renda tem sido associado a pior qualidade de vida.

Com o objetivo de favorecer a qualidade de vida das famílias de baixa renda a partir da melhoria de sua condição socioeconômica, foram implementados os Programas Sociais pelos governos de alguns estados da federação e pelo governo federal do Brasil. Entre estes programas, na presente pesquisa foram destacados o Renda Cidadã do Estado de São Paulo e o Bolsa Família de âmbito nacional.

A assistência social no Brasil foi instituída no âmbito da seguridade social a partir da Constituição de 1988 , na qual foram formalizados os direitos sociais básicos e o correspondente dever do Estado e da sociedade com relação às políticas sociais. Torres (2007) indica que o padrão de proteção social constituído pela Constituição se pautava na relação entre assistência e incapacidade de produzir e entre seguros sociais e pessoas com emprego estável. Neste sentido, Ferreira (1997) enfatiza que ficaram excluídos da proteção social os trabalhadores que não se enquadravam no sistema produtivo devido à modernização tecnológica e exigências do mercado de trabalho e os jovens à procura do primeiro emprego, pois ambos eram considerados aptos ao traba1ho, não tendo direito à assistência social, e, ao mesmo tempo, não estavam inseridos no mercado formal de trabalho, não possuindo, portanto, direito à seguridade social.

Frente a este contexto, a política de garantia de uma renda social mínima, entendida como um direito social de caráter não contributivo, representou uma estratégia para inserção desta população não coberta pela assis- tência e pelos seguros sociais na proteção social (Torres, 2007). Esta política, na forma de transferência de renda, tem início no Brasil na década de 1990 a partir de experiências descentralizadas e se efetiva como política nacional de mínimos sociais com a implementação do Programa Nacional de Renda Mínima em 2001 (Lei ${ }^{\circ}$ 10.219/01).

Segundo Fonseca (2001), os Programas de Transferência de Renda pressupõem que a insuficiência de renda das famílias obriga as crianças a entrarem precocemente no mercado de trabalho, abandonando a escola. Tal situação contribuiria para a perpetuação do ciclo da pobreza, pois dificultaria que as próximas gerações alcançassem postos de trabalho que exigem maiores qualificação e escolaridade. Desta forma, estes Programas têm como objetivo primordial o rompimento deste ciclo, o que pode ocorrer a partir do cumprimento das condicionalidades estabelecidas. Neste sentido, Dittrich (2008) enfatiza que o planejamento cultural deve pressupor que o comportamento precisa ser controlado por consequências de curto prazo para que as consequências benéficas à cultura sejam produzidas em longo prazo.

O Programa Renda Cidadã, formulado especificamente pelo Governo do Estado de São Paulo, por meio da Secretaria Estadual de Assistência e Desenvolvimento Social (SEADS), encontra-se regulamentado pela resolução SEADS 010, de 29 de junho de 2010. Podem ser beneficiárias deste Programa as famílias com renda per capita mensal de até meio salário mínimo nacional, comprovação de endereço e que possuem cadastro no Sistema Pró-Social, tendo prioridade as famílias chefiadas por mulheres.

Os objetivos do Programa Renda Cidadã se relacionam à promoção de ações complementares, à concessão de apoio financeiro temporário direto à família e à viabilização do acesso da população à rede de serviços. O valor mensal do benefício é de R $\$ 80,00$ por família, sendo concedido por um período de um ano, ou até o limite de 36 meses. As condicionalidades estabelecidas pelo Programa devem ser cumpridas pela família beneficiária para a sua permanência máxima e se relacionam à frequência escolar, saúde (controle da vacinação em crianças com idade até 6 anos) e participação das ações complementares (atividades que procuram favorecer o desenvolvimento da autonomia e a melhoria da qualidade de vida).

A SEADS destaca que o grupo de famílias que participa de programas de transferência de renda apresenta 
melhores condições de vida para aspectos como alimentação e educação, quando comparado às famílias não beneficiárias. Os participantes incluídos em serviços complementares, além da transferência de renda, apresentam condições de vida melhores que aqueles que apenas recebem o benefício (Pereira, 2009).

O Programa Bolsa Família, assim como o Renda Cidadã, consiste em um programa de transferência de renda com condicionalidades. Foi regulamentado no início de 2004 (Lei $\left.n^{\circ} 10.836 / 04\right)$ com a unificação dos procedimentos de gestão e execução das ações de transferência de renda do Governo Federal, como as do Programa Nacional de Renda Mínima vinculado à Educação (Bolsa Escola), do Programa Nacional de Acesso à Alimentação (PNAA), do Programa Nacional de Renda Mínima vinculado à Saúde (Bolsa Alimentação), do Programa Auxílio-Gás e do Cadastramento Único do Governo Federal.

De acordo com o Ministério de Desenvolvimento Social e Combate à Fome (MDS), podem ser beneficiadas pelo Programa Bolsa Família as famílias em situação de pobreza (renda per capita mensal entre R $\$ 70,01$ e R $\$ 140,00$ ) e de extrema pobreza (renda per capita mensal de até R\$ 70,00). Segundo o MDS, o Programa Bolsa Família atendia, no ano de 2012, mais de $13 \mathrm{mi}-$ lhões de famílias em todo território nacional de acordo com o perfil e tipos de benefícios, e tinha valor mínimo de R \$ 32,00. O Programa possui três eixos principais: transferência de renda, programas complementares e condicionalidades. A transferência de renda visa promover o alívio imediato da pobreza e as ações e programas complementares objetivam o desenvolvimento das famílias, de modo que os beneficiários consigam superar a situação de vulnerabilidade. As condicionalidades centram-se nas áreas da saúde, educação e assistência social.

Marques (2005), Paes-Sousa e Vaitsman (2007) e Tapajós e Quiroga (2010) abordaram pesquisas de avaliação do Programa Bolsa Família que se encontram publicadas na série Caderno de Estudos disponibilizada no site do MDS. Estas pesquisas, segundos os autores, têm demonstrado melhoria da qualidade de vida dos beneficiários do Programa devido à possibilidade de aquisição de gêneros alimentícios com o benefício, menor quantidade de partos prematuros, maior proporção de crianças beneficiárias consideradas nutridas e aumento dos índices de vacinação em dia e da frequência escolar de crianças de 6 a 17 anos.

Por outro lado, Alberini (2010), Lund et al. (2011) e
Magalhães, Nogueira, Coelho e Bocca (2011) enfatizam que a transferência condicionada de renda por meio dos Programas Sociais não são suficientes para promoção de melhores índices de saúde e qualidade de vida entre a população beneficiária. Os autores argumentam que existe necessidade de melhor articulação entre as diferentes políticas (saúde, educação e assistência social).

Nesta direção, a presente pesquisa teve como objetivo principal identificar a prevalência de stress, depressão e percepção da qualidade de vida em beneficiários de programas de transferência de renda e como objetivos específicos assinalar aspectos sociodemográficos da amostra, determinar sintomas e fases de stress predominantes e averiguar relações entre stress, depressão e qualidade de vida.

\section{MÉTODO}

\section{Participantes}

Participaram da pesquisa 198 beneficiários dos Programas de Transferência de Renda "Renda Cidadã" e "Bolsa Família" moradoras de uma cidade localizada no interior do estado de São Paulo. Os critérios de inclusão foram ter mais de 18 anos de idade, estar inscrito em um Centro de Referência da Assistência Social (CRAS) do município e frequentar reuniões sistemáticas realizadas neste Centro. O critério de exclusão foi não conseguir ler e responder aos instrumentos. A amostra constituiu-se por 36 beneficiários do Programa Bolsa Família, 85 do Renda Cidadã e 77 que participavam dos dois Programas, visto que a mesma pessoa poderia receber os dois benefícios ao mesmo tempo. $\mathrm{Na}$ cidade onde ocorreu a coleta dos dados o número total de famílias beneficiárias dos Programas de Transferência de Renda, no período da coleta, era em torno de 1.020 famílias inclú́das no Renda Cidadã e 8.800 no Bolsa Família.

A coleta dos dados ocorreu nos CRAS, os quais se constituem em unidades estatais que funcionam como "porta de entrada" da rede de serviços socioassistenciais do território, onde são prestadas informações e orientações para a população de sua área de abrangência. No município de coleta de dados havia um total de nove CRAS, distribuídos nas áreas de maior vulnerabilidade social.

\section{Instrumentos}

Foram utilizados os seguintes intrumentos: um 
Questionário Sociodemográfico, o Inventário de Sintomas de Stress para Adultos de Lipp - ISSL (Lipp, 2000), o Inventário de Depressão de Beck - BDI (Cunha, 2001) e o Instrumento Abreviado de Avaliação da Qualidade de Vida - WHOQOL-Bref (The Whoqol Group Brazil, 1998).

O ISSL é um instrumento feito no Brasil e validado em amostra brasileira que visa identificar se o sujeito apresenta sintomas de stress, o tipo predominante (físico ou psicológico) e a fase em que se encontra (Alerta, Resistência, Quase-Exaustão ou Exaustão). O instrumento contém três quadros referentes às quatro fases do stress. No primeiro quadro deve-se assinalar os sintomas vivenciados nas últimas 24 horas. O segundo quadro indica os sintomas experimentados na última semana. No terceiro quadro devem ser assinalados os sintomas experimentados no último mês. Para avaliar se uma pessoa apresenta sintomas significativos de stress, verifica-se o escore bruto total de respostas por quadro, sendo que é considerada como tendo stress aquela que no Quadro 1 apresenta a soma de sintomas psicológicos e físicos superior a 6 ou que no Quadro 2 apresenta soma superior a 3 ou que no Quadro 3 apresenta soma maior que 8. Para determinar em que fase de stress o indivíduo se encontra verificam-se as porcentagens correspondentes aos resultados brutos. Destaca-se que no Quadro 2 porcentagens de até 50 indicam fase de resistência e acima de 50 indicam fase de quase-exaustão. Para determinar a sintomatologia predominante, verifica-se a porcentagem correspondente a fase de stress em que a pessoa se encontra.

O BDI consiste em um questionário de autoavaliação com 21 itens de múltipla escolha, cada um com quatro alternativas que subentendem graus crescentes de gravidade da depressão. Seus itens relacionam-se a: Tristeza; Pessimismo; Sentimento de fracasso; Insatisfação; Culpa; Punição; Autoaversão; Autoacusações; Ideias suicidas; Choro; Irritabilidade; Retraimento social; Indecisão; Mudança na autoimagem; Dificuldades de tralhar; Insônia; Fatigabilidade; Perda de apetite; Perda de peso; Preocupações somáticas; Perda da libido. Cada resposta recebe um valor de 0 a 3 . O escore total é o resultado da soma dos escores individuais dos itens. Quando a soma das respostas fica entre 0 e 13 diz-se que é um indicativo de depressão mínima, entre 14 e 19 depressão leve, entre 20 e 28 depressão moderada e entre 29 e 63 depressão severa. Tal instrumento foi validado em amostras populacionais brasileiras por Gorenstein e Andrade em 1998 e por Cunha em 2001.
O WHOQOL-Bref foi desenvolvido pela OMS numa perspectiva transcultural, tendo sido abreviado a partir do WHOQOL-100, o qual apresenta quatro questões gerais sobre a qualidade de vida e mais 96 divididas em 24 facetas: dor e desconforto; energia e fadiga; sono e repouso; sentimentos positivos; pensar, aprender, memória e concentração; autoestima; imagem corporal e aparência; sentimentos negativos; mobilidade; atividades da vida cotidiana; dependência de medicação ou de tratamentos; capacidade de trabalho; relações pessoais; suporte (apoio) social; atividade sexual; segurança física e proteção; ambiente no lar; recursos financeiros; cuidados de saúde e sociais; disponibilidade e qualidade; oportunidades de adquirir novas informações e habilidades; participação em, e oportunidades de recreação/lazer; ambiente físico (poluição, ruído, trânsito, clima); transporte; espiritualidade, religiosidade, crenças pessoais. A versão abreviada contém 26 questões, sendo que duas se referem à percepção do indivíduo em relação à qualidade de vida e as demais representam cada uma das 24 facetas, as quais se encontram divididas em quatro domínios: Físico, Psicológico, Relações Sociais e Meio Ambiente. A pontuação dos escores deve ser realizada utilizando-se o programa estatístico SPSS com a sintaxe do WHOQOL-bref. O critério de seleção das questões que compõem o instrumento abreviado foi tanto psicométrico, como conceitual.

\section{Procedimento}

Inicialmente foi encaminhado ofício à Secretaria responsável pela gestão dos Programas Renda Cidadã e Bolsa Família de uma cidade do interior do estado de São Paulo, solicitando autorização para utilização dos dados destes Programas para fins de pesquisa. Fez-se contato com os CRAS para agendamento da coleta de dados de acordo com a disponibilidade do local. Os beneficiários que aceitaram participar da pesquisa assinaram o Termo de Consentimento Livre e Esclarecido em duas vias. Todos os cuidados éticos foram tomados segundo o Regulamento do Sistema Único de Saúde 2048-09 e o projeto foi aprovado sob $\mathrm{n}^{\circ}$ 8193/46/01/11 pelo Comitê de Ética em Pesquisa da Universidade. A coleta de dados ocorreu em grupos e o número de pessoas por grupo variou de 7 a 20 , sendo montados 16 grupos.

Em todos os grupos o procedimento seguiu a mesma ordem: primeiramente o ISSL, depois o BDI, em seguida o WHOQOL-Bref e, por último, o questionário sociodemográfico. Os instrumentos (instruções e itens) 
e o questionário sociodemográfico foram lidos em voz alta pelo pesquisador principal da área de psicologia e cada participante os respondeu individualmente. $\mathrm{Na}$ análise quantitativa, as informações coletadas foram sintetizadas por meio das funções da estatística descritiva. Para isto, foram utilizados os testes estatísticos " $\mathrm{t}$ " de Student, ANOVA e correlação de Pearson.

\section{RESULTADOS}

A faixa etária da amostra pesquisada se distribuiu entre 18 e 74 anos. A maioria constituiu-se como sendo do sexo feminino. A renda per capita variou entre $\mathrm{R} \$ 0,00$ e $\mathrm{R} \$ 622,00$. Quanto à moradia, $88 \%$ apontaram residir na zona urbana. Os dados referentes às características sociodemográficas da amostra se encontram sintetizados na Tabela 1.

$\mathrm{Na}$ investigação da variável stress, verificou-se que $58 \%$ apresentaram stress, destes, $68 \%$ estavam na Fase de Resistência, 24\% na Fase de Quase-Exaustão, 7\% na Fase de Exaustão e 1\% na Fase de Alerta, sendo que $47 \%$ exibiram predominância de sintomas psicológicos, $38 \%$ de sintomas físicos e 15\% referiram sintomas físicos e psicológicos. Quanto aos indicativos de depressão, 34\% apresentaram indicativo de depressão mínima, $27 \%$ de depressão moderada, $25 \%$ de depressão leve e $14 \%$ de depressão grave. O índice médio de depressão obtido foi de 19,2 , sendo que o desvio padrão foi de 12,9 e a mediana 17 num intervalo de confiança de 95\%. No que se refere ao Índice Global da Qualidade de Vida, a média obtida pelos participantes foi de 51,3. Nos domínios referentes à qualidade de vida as médias foram de 54,9 (Psicológico), 54,1 (Físico), 53,4 (Relações Sociais) e 43,0 (Meio Ambiente), em uma escala que varia de 0 a 100 .

As variáveis investigadas foram analisadas em relação aos dados sociodemográficos, sendo que foram obtidas relações estatisticamente significativas nos seguintes aspectos: os participantes que cursaram Ensino Médio apresentaram maior índice médio de qualidade de vida no domínio físico $(64,44)$, seguidos por aqueles que tinham entre $5^{\mathrm{a}}$ e $8^{\mathrm{a}}$ séries $(54,24)$, Ensino Técnico $(48,83)$ e entre $1^{\mathrm{a}}$ e $4^{\mathrm{a}}$ séries $(48,67)$, sendo $\mathrm{p}=0,001$; participantes moradores da zona rural obtiveram maior índice médio de percepção da qualidade de vida no que se refere ao domínio meio ambiente $(47,97)$, do que os moradores da zona urbana $(42,35)$, sendo $p=0,053$. Relação significativa limítrofe $(p=0,064)$ foi percebida entre escolaridade e o índice médio referente ao domínio meio ambiente de avalia-
Tabela 1

Descrição das variáveis sociodemográficas da amostra de beneficiários de Programas de Transferência de Renda

\begin{tabular}{|c|c|c|}
\hline Variáveis & Categorias & $\begin{array}{l}\text { Número de } \\
\text { Participantes }\end{array}$ \\
\hline \multirow{3}{*}{ Idade } & 18 a 30 anos & 55 \\
\hline & 31 a 50 anos & 68 \\
\hline & 51 a 74 anos & 75 \\
\hline \multirow{2}{*}{ Sexo } & Feminino & 183 \\
\hline & Masculino & 15 \\
\hline \multirow{4}{*}{ Estado Civil } & Solteiro & 81 \\
\hline & União Estável & 73 \\
\hline & Separado & 35 \\
\hline & Viúvo & 09 \\
\hline \multirow{4}{*}{ Escolaridade } & $1^{\mathrm{a}} \mathrm{a} 4^{\mathrm{a}} \mathrm{EF}$ & 84 \\
\hline & $5^{\mathrm{a}}$ a $8^{\mathrm{a}} \mathrm{EF}$ & 67 \\
\hline & $1^{\circ}$ ao $3^{\circ} \mathrm{EM}$ & 44 \\
\hline & Ensino Técnico & 03 \\
\hline \multirow{3}{*}{$\begin{array}{l}\text { Renda Per } \\
\text { Capita }\end{array}$} & Até $\mathrm{R} \$ 70,00$ & 61 \\
\hline & De $R \$ 70,01$ a $R \$ 140,00$ & 63 \\
\hline & De $R \$ 140,01$ a $R \$ 622,00$ & 74 \\
\hline \multirow{5}{*}{ Ocupação } & Informal & 81 \\
\hline & Formal & 06 \\
\hline & Desempregado & 18 \\
\hline & Do lar & 89 \\
\hline & Aposentado & 04 \\
\hline \multirow{2}{*}{ Residência } & Zona Urbana & 175 \\
\hline & Zona Rural & 23 \\
\hline \multirow{3}{*}{ Beneficio } & $\mathrm{BF}$ & 36 \\
\hline & $\mathrm{RC}$ & 85 \\
\hline & $B F+R C$ & 77 \\
\hline
\end{tabular}

ção da qualidade de vida, sendo que os participantes que cursaram Ensino Técnico apresentaram maior índice médio $(48,96)$, seguidos por aqueles que possuem Ensino Médio $(47,75), 4^{\mathrm{a}}$ série $(42,53)$ e entre $5^{\mathrm{a}}$ e $8^{\mathrm{a}}$ séries $(40,21)$.

A análise correlacional demonstrou-se significativa, sendo que stress e depressão correlacionaram-se positivamente e stress e qualidade de vida e depressão e qualidade de vida correlacionaram-se de forma negativa. Tais dados se encontram destacados na Tabela 2.

As correlações positivas indicam relação direta entre as variáveis e as negativas sinalizam relação inversa. Os valores de $r$ demonstram a correlação e variam de 1 a -1 , sendo estes as correlações máximas. Para analisar uma correlação deve-se levar em consideração o nível de significância $(p)$, a direção (+ou-) e a magnitude. 
Tabela 2

Correlação entre Stress, Depressão e Qualidade de Vida na população inserida em Programas de Transferência de Renda

\begin{tabular}{lcccccccc}
\hline & & Stress & Depressão & $\begin{array}{c}\text { QV } \\
\text { Global }\end{array}$ & $\begin{array}{c}\text { QV- } \\
\text { Físico }\end{array}$ & $\begin{array}{c}\text { QV - } \\
\text { Psicológico }\end{array}$ & $\begin{array}{c}\text { QV - Relações } \\
\text { Sociais }\end{array}$ & $\begin{array}{c}\text { QV - Meio } \\
\text { Ambiente }\end{array}$ \\
\hline \multirow{2}{*}{ Stress } & $r$ & 1 & 0,49 & $-0,19$ & $-0,36$ & $-0,35$ & $-0,27$ & $-0,28$ \\
& $p$ & & 0,001 & 0,035 & 0,001 & 0,001 & 0,003 & 0,002 \\
\hline \multirow{2}{*}{ Depressão } & $r$ & 0,49 & 1 & $-0,25$ & $-0,50$ & $-0,61$ & $-0,56$ & $-0,39$ \\
& $p$ & 0,001 & & 0,001 & 0,001 & 0,001 & 0,001 & 0,001 \\
\hline \multirow{2}{*}{ Qualidade de Vida } & $r$ & $-0,19$ & $-0,25$ & 1 & 0,41 & 0,33 & 0,31 & 0,35 \\
Global & $p$ & 0,035 & 0,001 & & 0,001 & 0,001 & 0,001 & 0,001 \\
\multirow{2}{*}{ Domínio Físico } & $r$ & $-0,36$ & $-0,50$ & 0,41 & 1 & 0,66 & 0,51 & 0,62 \\
& $p$ & 0,001 & 0,001 & 0,001 & & 0,001 & 0,001 & 0,001 \\
\hline \multirow{2}{*}{ Domínio Psicológico } & $r$ & $-0,35$ & $-0,61$ & 0,33 & 0,66 & 1 & 0,59 & 0,62 \\
& $p$ & 0,001 & 0,001 & 0,001 & 0,001 & & 0,001 & 0,001 \\
\hline \multirow{2}{*}{ Relações Sociais } & $r$ & $-0,27$ & $-0,56$ & 0,31 & 0,51 & 0,59 & 1 & 0,53 \\
& $p$ & 0,001 & 0,001 & 0,001 & 0,001 & 0,001 & & 0,001 \\
\hline \multirow{2}{*}{ Meio Ambiente } & $r$ & $-0,28$ & $-0,39$ & 0,35 & 0,62 & 0,62 & 0,53 & 1 \\
& $p$ & 0,002 & 0,001 & 0,001 & 0,001 & 0,001 & 0,001 & \\
\hline
\end{tabular}

Observou-se que quanto mais avançado o processo de stress, maior foi o indicativo de depressão e menor o índice de percepção de qualidade de vida obtidos.

\section{DISCUSSÃO}

Alguns estudos têm colocado a relação entre desigualdade social e saúde mental, enfatizando que as populações em condição de pobreza podem estar mais suscetíveis ao desenvolvimento de problemas de saúde, como stress, transtornos mentais e a piores índices de qualidade de vida (Alberini, 2010; Hernández et al., 2007; Kaplan et al., 2013; Lund et al., 2011; Magalhães et al., 2011; Probst et al., 2005). Estas pesquisas demonstram a importância da realização de investigações com a população de baixa renda.

No presente estudo, os resultados obtidos em relação à variável stress foram semelhantes aos dados encontrados por Lipp (2000) e Ramos e Ferreira (2011) no que diz respeito à sua prevalência e à fase predominante (resistência). Quanto aos dados referentes à depressão, considerando-se que o escore que possivelmente aponta para o desenvolvimento de um transtorno depressivo, segundo Cunha (2001), é de 20 ou mais, verificou-se que $41 \%$ da amostra apresentou indicativo sugestivo de depressão (depressão moderada ou severa). Este percentual de participantes com escore sugestivo de depressão está acima do encontrado em algumas pesquisas (Cunha et al., 2012; Nunes \& Santos, 2010), além de também estar acima do demonstrado pela po- pulação brasileira em geral segundo dados da OMS, que apontou prevalência de $10,4 \%$ para o ano de 2009 (Bromet et al., 2011). Cogita-se que isto ocorreu porque a amostra possui muitas das características que, conforme enfatizam Lund et al. (2011), Máximo (2010) e Santos (2011), relacionam-se aos fatores de risco para desenvolvimento da depressão, como condição socioeconômica menos favorecida, baixa escolaridade e situação de desemprego/sem trabalho.

Os índices médios de percepção da qualidade de vida obtidos foram menores que os observados em outros estudos, como o de Nunes e Santos (2010) e o de Pereira et al. (2011). Foi percebida diferença significativa em relação à escolaridade no domínio físico do WHOQOL-Bref, sendo que os participantes com Ensino Médio exibiram o índice médio mais alto e os com escolaridade entre $1^{\mathrm{a}}$ e $4^{\mathrm{a}}$ séries, o menor. Observaramse diferenças significativas limítrofes quanto à condição socioeconômica e moradia no domínio meio ambiente. A condição socioeconômica de baixa renda tem sido constantemente relacionada a escores de qualidade de vida menores, conforme exposto por Nunes e Santos (2010) e Pereira et al. (2011). Este fator pode estar associado aos baixos índices de percepção da qualidade de vida obtidos neste trabalho, pois todos os participantes se encontravam nesta condição.

Observa-se que foram encontradas poucas diferenças significativas em relação às características sociodemográficas e os dados obtidos por meio dos 
instrumentos. Isto pode ter ocorrido, conforme apontam Chapela et al. (2009), devido à uniformidade da amostra quanto à condição socioeconômica, que, segundo estes autores, deve estar enquadrada em determinado perfil que a torna elegível para inserção nos Programas de Transferência de Renda.

Os estudos de Blay e Marchesoni (2011) e Nunes e Santos (2010), embora com populações diferentes, demonstraram a mesma correlação entre depressão e qualidade de vida observadas nesta pesquisa. Índices maiores de depressão parecem contribuir para baixos escores referentes à percepção da qualidade de vida.

Quanto aos Programas de Transferência de Renda abordados, observa-se que tanto a SEADS, responsável pelo Programa Renda Cidadã, como o MDS, responsável pelo Programa Bolsa Família, têm procurado avaliar o impacto dos Programas na vida dos beneficiários (Marques, 2005; Paes-Sousa \& Vaitsman, 2007; pereira, 2009; Tapajós \& Quiroga, 2010). Este impacto, frequentemente, tem sido destacado em termos de melhora do poder de consumo e cumprimento das condicionalidades dos Programas.

Tem sido observada a promoção de alívio imediato da situação de pobreza e extrema pobreza por meio da transferência de renda, conforme objetivos propostos na implantação dos Programas de Transferência de Renda, segundo dados de pesquisas realizadas pela SEADS e pelo MDS (Marques, 2005; Paes-Sousa \& Vaitsman, 2007; Pereira, 2009; Tapajós \& Quiroga, 2010). Essa melhoria tem sido avaliada em termos do aumento do consumo de itens variados e do consequente impacto socioeconômico deste aumento. Porém, os demais objetivos a que os Programas se propõem (Renda Cidadã favorecer o desenvolvimento da autonomia dos beneficiários e a melhoria da sua qualidade de vida; Bolsa Família - promover o desenvolvimento das famílias, de modo que os beneficiários consigam superar a situação de vulnerabilidade) parecem carecer de operacionalização, o que tem dificultado a sua avaliação.

\section{CONSIDERAÇÕES FINAIS}

É cada vez maior o número de psicólogos que atuam na área de assistência social. Diante disso, mostra-se de grande relevância a realização de estudos com famílias de baixa renda que possam contribuir para melhoria dos serviços prestados por este profissional. A presente pesquisa teve a pretensão de iniciar este processo a fim de subsidiar os profissionais que atuam com esta popula- ção a desenvolverem ações que efetivamente resultem na melhoria de qualidade de vida.

Na literatura é consenso que a situação socioeconômica de baixa renda é fator de risco para desenvolvimento de piores condições de saúde mental. Percebe-se que a transferência de renda ocasionada pelos Programas Sociais impacta positivamente a vida dos beneficiários, que têm apresentado maiores poder de consumo e acesso aos serviços nas áreas de saúde e educação. No entanto, apesar da melhoria nestes aspectos, os beneficiários participantes deste estudo ainda apresentam altos escores indicativos de transtorno depressivo $\mathrm{e}$ baixos índices de percepção da qualidade de vida. Porém, somente estudos longitudinais e comparativos com amostras controle poderiam efetivamente dar suporte a este resultado.

Enfatiza-se a importância da realização de pesquisas que abarquem a população de baixa renda não inseridas nestes Programas, visto que não possuem o impacto da transferência de renda nas suas condições de vida e podem apresentar piores resultados relacionados à saúde mental. Coloca-se, também, a relevância de estudos longitudinais que visem avaliar as condições de vida dos beneficiários incluídos em Programas de Transferência na inserção e após a passagem do tempo, em diferentes momentos do Programa, no sentido de verificar se houve mudança na qualidade de vida após se tornarem beneficiários.

Coloca-se como limitação do estudo a seleção da amostra, que se caracterizou por aqueles beneficiários que frequentavam os CRAS, constituindo-se em uma amostra de conveniência. Sugere-se que novos estudos consigam superar esse viés.

Por último, enfatiza-se que os dados obtidos em relação ao stress, depressão e qualidade de vida na população brasileira em vulnerabilidade social podem abrir caminho para novas pesquisas, uma vez que investigações com essa temática nesta população têm sido pouco observadas na revisão de literatura.

\section{REFERÊNCIAS}

Alberini, M. (2010). Nos limites do viver e do sobreviver: o programa Bolsa Família, modos de vida e desenvolvimento social no contexto urbano. Dissertação de Mestrado, Faculdade de Saúde Pública - Universidade de São Paulo, São Paulo.

Alchieri, J. C., \& Cruz, R. M. (org). (2004). Estresse: conceitos, métodos, medidas e possibilidades de intervenção, São Paulo, SP: Casa do Psicólogo, 2004. 
Baptista, M. N., Vargas, J. F., \& Baptista, A. S. D. (2008). Depressão e qualidade de vida em uma amostra brasileira de obesos mórbidos. Avaliação Psicológica, 7 (2), 235 127.

Blay, S. L., \& Marchesoni, M. S. M. (2011). Association among physical, psychiatric and socioeconomic conditions and WHOQOL-Bref scores. Caderno de Saúde Pública, 27 (4), 677-686.

Bromet, E., Andrade, L .H., Hwang, I., Sampson, N. A., Alonso, J., Girolamo, G.,... Kessler, R. C. (2011). Crossnational epidemiology of DSM-IV major depressive episode. BMC Medicine, 9 (90), 1-16.

Chapela, I. B., Daniel, V. E. V., Espinoza, B. S. M., Rojo, M. M. T., \& rodríguez, A. S. (2009). Depressive symptoms among poor older adults in Mexico: prevalence and associated factors. Revista Panamericana de Salud Pública, 26 (1), 70-77.

Cunha, J. A. (2001). Manual da versão em português das Escalas Beck. São Paulo: Casa do Psicólogo.

Cunha, R. V., Basco, G. A. N., \& Del Duca, G. F. (2012). Prevalência de depressão e fatores associados em comunidade de baixa renda de Porto Alegre, Rio Grande do Sul. Revista Brasileira de Epidemiologia, 15 (5), 346354.

Dantas, M. A., Brito, D. V. C., Rodrigues, P. B., \& Maciente, T. S. (2010). Avaliação de estresse em policiais militares. Psicologia: teoria e prática, 12 (3), 66-77.

Decreto-Lei n ${ }^{\circ} 10.219$ de 11 de abril de 2001. Diário Oficial [da] República Federativa do Brasil, Poder Executivo, Brasília, DF.

Decreto-Lei $\mathrm{n}^{\circ} 10.836$ de 09 de janeiro de 2004. Diário Oficial [da] República Federativa do Brasil, Poder Executivo, Brasília, DF.

Dittrich, A. (2008). Sobrevivência ou colapso? B. F. Skinner, J. M. Diamond e o Destino das Culturas. Psicologia: Reflexão e Crítica, 21 (2), 252-260.

Dressler, W. W., \& Balieiro, M. C. (2002). Cultura e Estresse Psicológico. Paideia, 12 (22), 5-18.

Ferreira, A. A. (1997). Gestão Empresarial: de Taylor aos nossos dias: evolução e tendências da moderna administração de empresas. São Paulo: Pioneira.

Fonseca, J. P. (2001). Projeto pedagógico: processo e produto na construção coletiva do sucesso escolar. Jornal APASE Suplemento Pedagógico, São Paulo, 1-4.

Gordia, A. P., Quadros, T. M. B., Campos, W., \& Vilela-Junior, G. B. (2009). Qualidade de vida de adolescentes da rede particular de ensino: comparação entre gêneros. Revista Brasileira de Qualidade de Vida, 1 (2), 16-24.

Gorenstein, C., \& Andrade, L. H. S. G. (1998). Inventário de Depressão de Beck: propriedades psicométricas da versão em português, Revista Brasileira de Psiquiatria Clínica, 25 (5), 245-250.

Hernández, L. O., Moreno, S. L., \& Borges, G. (2007). Desigualdad socioeconómica y salud mental: revisión de la literatura latino-americana. Caderno de Saúde Pública, 23 (6), 1255-1272.

Kaplan, S. A., Madden, V. P., Mijanovich, T., \& Purcaro, E. (2013). The perception of stress and its impact on health in poor communities. J Community Health, 32, 142-149.
Lipp, M. N. E. (org). (1996). Pesquisa sobre stress no Brasil: saúde, ocupações e grupos de risco (2 $2^{\mathrm{a}} \mathrm{d}$.). São Paulo, SP: Papirus Editora.

Lipp, M. N. E. (2000) Manual do inventário de sintomas de stress para adultos de Lipp (ISSL), ( $2^{\mathrm{a}}$ ed.). São Paulo: Casa do Psicólogo.

Lund, C., Silva, M., Plagerson, S., Cooper, S., Chisholm, D., Das, J.,... Patel, V. (2011). Poverty and mental disorders: breaking the cycle in low-income and middle-income countries. Lancet, 378, 1502-1514.

Marques, R. M. (org) (2005). Cadernos de Estudos. Desenvolvimento Social em Debate: síntese das pesquisas de avaliação de programas sociais do MDS, 1, Brasília: Ministério do Desenvolvimento Social e Combate à Fome. .

Magalhães, R., Coelho, A. V., Nogueira, M. F., \& Bocca, C. (2011). Intersetorialidade, Convergência e Sustentabilidade: Desafios do Programa Bolsa Família em Manguinhos, RJ. Ciência e Saúde Coletiva, 16 (11), 4443-4453.

Martin, D., Quirino, J., \& Mari, J. (2007). Depressão entre mulheres de periferia de São Paulo. Revista de Saúde Pública 41 (4), 591-597.

Mastropietro, A. P., Oliveira-Cardoso, E. A., Simões, B. P., Voltarelli, J. C., \& Santos, M. A. (2010). Relação entre renda, trabalho e qualidade de vida em pacientes submetidos ao transplante de medula óssea. Revista Brasileira de Hematologia e Hemoterapia, 32 (2), 102-107.

Máximo, G. C. (2010). Aspectos sociodemográficos da depressão e utilização de serviços de saúde no Brasil. Tese do Curso de Pós-Graduação em Demografia do Centro de Desenvolvimento e Planejamento Regional - Universidade Federal de Minas Gerais, Belo Horizonte.

Ministério de Desenvolvimento Social e Combate à Fome (2013). XXX [em linha]. Ministério de Desenvolvimento Social e Combate à Fome (MDS) Web site.

Munhoz, T., Nunes, B. P., Wehrmeister, F. C., Santos, I. S., \& Matijasevich (2016). A nationwide population-based study of depression in Brazil. Journal of Affective Disorders, 192, 226-233.

Nunes, M. A. F., \& Santos, M. A. (2010). Depressão e qualidade de vida em mães de crianças com transtornos invasivos do desenvolvimento. Revista Latino Americana de Enfermagem, 18 (1), 1-9.

Paes-Sousa, R., \& Vaitsman, R. (org) (2007). Cadernos de Estudos. Desenvolvimento Social em Debate: síntese das pesquisas de avaliação de programas sociais do MDS, 5, Brasília: Ministério de Desenvolvimento Social e Combate à Fome.

Pafaro, R. C., \& Martino, M. M. F. (2004). Estudo do estresse do enfermeiro com dupla jornada de trabalho em um hospital de oncologia pediátrica de Campinas. Revista da Escola de Enfermagem - USP, 38 (2), 152-160.

Pereira, F. S. (2009). Renda Cidadã - Programa de Transferência Condicionada de Renda do Governo do Estado de São Paulo. In II Congresso Consad de Gestão Pública, Brasília. Anais Eletrônicos. Painel.

Pereira, K. C. R., Alvarez, A. M., \& Traebert, J. L. (2011). Contribuição das condições sociodemográficas para a percepção da qualidade de vida em idosos. Revista Brasileira de Geriatria e Gerontologia, 14 (1), 85-95. 
Prigerson, H. G., Maciejewski, P. K., \& Rosenback, R. A. (2002). Population attributable fractions of psychiatric disorders and behavioral outcomes associated with combat exposure among US men. American Journal of Public Health, 92 (1), 59-63.

Probst, J. C., Laditka, S., Moore, C. G., Harun, N., \& Powell, M. P. (2005). Depression in Rural Populations: prevalence, effects on life quality, and treatment-seeking behavior. Rural Health Research Center, 1-4.

Ramos, L., \& Ferreira, E. A. P. (2011). Fatores emocionais, qualidade de vida e adesão ao tratamento em adultos com diabetes tipo 2. Revista Brasileira de Crescimento e Desenvolvimento Humano, 21 (3), 867-877.

Resolução $\mathrm{n}^{\circ} 10$ de 29 de junho de 2010. Diário Oficial do Estado de São Paulo, Poder Executivo, São Paulo, v. 120, n. 129, p. 1-16. Secretaria de Assistência e Desenvolvimento Social.

Santos, P. R. (2011). Depression and quality of life hemodialysis patients living in a poor region of Brazil. Revista Brasileira de Psiquiatria, 33 (4), 332-337.

Sparrenberger, F., Santos, I., \& Lima, R. C. (2004). Associação de eventos de vida produtores de estresse e mal estar psicológico: um estudo de base populacional. Caderno de Saúde Pública, (Rio de Janeiro), 20 (1), 249-258.
Tapajós L., \& Quiroga, J. (org) (2010). Cadernos de Estudos. Desenvolvimento Social em Debate: síntese das pesquisas de avaliação de programas sociais do MDS, 13, Brasília: Ministério de Desenvolvimento Social e Combate à Fome.

The Whoqol Group. (1995). The World Health Organization quality of life assessment (WHOQOL): position paper from the World Health Organization [em linha]. Social Science and Medicine Website.

The Whoqol Group Brazil (coord.) (1998). WHOQOL Abreviado: versão em português.

Torres, J. C. (2007). O SUAS e a universalização da renda social mínima no Brasil. In Revista Serviço Social e Sociedade. Política Social Desafios para o Serviço Social (43-57). São Paulo: Cortez.

Notas:

1 Cursa Doutorado em Fonoaudiologia - Processos e Distúrbios da Comunicação - pela Faculdade de Odontologia de Bauru - USP. Psicóloga no Hospital de Reabilitação de Anomalias Craniofaciais da Universidade de São Paulo. Contato: marianicribas@yahoo.com.br

2 Possui Doutorado em Psicologia pela Pontifícia Universidade Católica de Campinas. Especialista em Psicologia Clínica Docente da Universidade Estadual Paulista Júlio de Mesquita Filho - UNESP e membro do programa de pós-graduação em Psicologia do Desenvolvimento e Aprendizagem UNESP-Bauru. Contato: scalais@fc.unesp.br

3 Doutorado e Pós doutorado em Psicologia pela Universidade São Francisco. Professor da Universidade Estadual Paulista Júlio de Mesquita Filho (UNESP). Contato: hfcardoso@fc.unesp.br 